\title{
MECHANICAL RESPONSES OF SMOOTH MUSCLE TO ELECTRIC STIMULI
}

\author{
GORO KAWABATA ${ }^{1}$ \\ Department of Physiology (1st Division), \\ Kyoto University, Kyoto
}

Smooth muscle exhibits various types of shortening in response to mechanical, thermal, chemical or electric stimuli. These responses are usually designated tone, contracture, tonic or phasic contractions. The meaning of these terms, though frequently used, is not always clear, as the confused usage of tone and contracture is often encountered in the literature. No doubt the confusion originates in the lack of our knowledge of the fundamental mechanism which underlies the mechanical responses in smooth muscle. As to electric stimuli, Winton (10) and Singh (9) pointed out that the retractor of the byssus of mytilus responds to alternating currents with phasic contractions, while it responds to direct currents with prolonged tonic contractions. These findings seem to be important, not only because they indicate the presence of at least two fundamentally different contractile processes in smooth muscle, but also because they offer a clue to possible classification of contractile mechanisms, which might result phenomenologically in various kinds of shortening. The present communication deals with the mechanical responses of toad's bladder to electric stimuli with the purpose of elucidating contractile mechanism in general and its relation to excitability in smooth muscle.

\section{METHODS}

Almost all experiments were carried out on muscle strips cut out of the toad's bladder. In a few cases muscle strips from toad's stomach were used for comparison. The muscle strip was hung vertically in Ringer's fluid and the mechanical responses were registered on a kymograph by an isotonic lever, which was attatched by means of a serrefine to the upper end of the preparation. The serrefine was made of chlorided silver and served as an electrode in electric stimulation (fig. 1). The other electrode, which was also a chlorided silver wire, was inserted in the saline at the bottom of the vessel. Just before stimulation the saline was withdrawn so that the whole preparation except the lower end, which still remained in contact with the fluid, was exposed to air, and the stimulating currents were made to flow through the whole length of the preparation. In case of nerve stimulation, the bladder was suspended in situ and the peripheral cut end of the vesical nerve of one side was stimulated in

1 川端五郎

Received for publication October 26, 1950. 


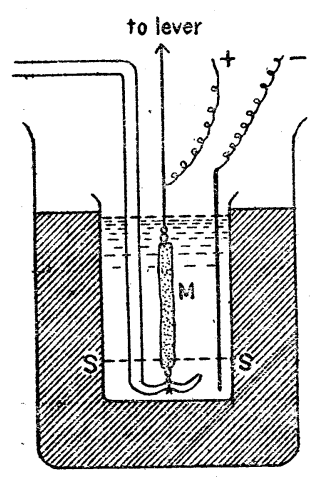

Fig. 1. Diagram illustrating the method of delivering an electric current to a muscle strip. $M$ : Muscle strip. Ringer's fluid in the beaker was withdrawn to the level $S S$ previous to each electric stimulation.

air by the chlorided silver electrodes.

Electric currents used for direct stimulation were single and repetitive induction shocks, direct current, alternating current and unidirectional pulse current obtained by passing an alternating current through a vacuum tube. As the rheobase was very low, a non-inductive resistance ( 1 megohm), so high that the resistance of the tissue could be neglected, was inserted in series to preparation and the current intensity was calculated from the applied voltage and resistance. The induction currents were applied directly without shunt circuit and resistance.

The source of D.C. was a battery, while that of A.C. was a street line (60 cycle), that was connected to a potentiometer circuit through a transformer, which served to insulate the stimulating circuit including the preparation, from the earth. The intensity of alternating current was measured by cathode-ray oscilloscope. Alternating currents of $60 \sim 1,000$ cycles were also obtained by an oscillator (Matzuda, Type C), and sometimes used for stimulation after they made unidirectional pulse currents.

\section{RESULTS}

1. Responses of bladder to nerve stimulation and responses of muscle strip to direct stimulation with alternating current

When a faradic current of relatively short duration $(3 \sim 5 \mathrm{sec}$.) was delivered

Fig. 2. The height of contractions in relation to current intensity. Abscissa : current intensity (coil distance; effective intensity). Ordinate: contraction height. Circles : direct stimulation of muscle strip with A.C. Crosses: stimulation of vesical nerve with faradic current.

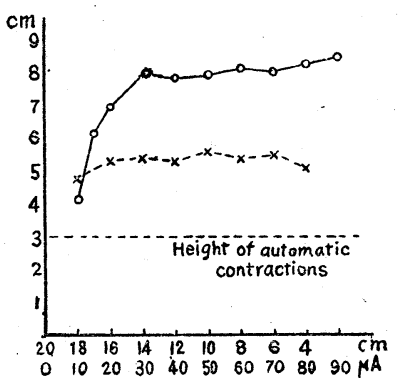


to the vesical nerve, the bladder responded to it with a short phasic contraction. The contraction was augmented in accordance with an increase in current intensity, until it attained a certain maximal height, which thereafter remained nearly constant notwithstanding the further increase in current intensity, provided that the current duration was unaltered (fig. 2). The submaximal contractions seemed to be due to submaximal excitation of vesical nerve, which in turn resulted in partial excitation of contractile elements in the bladder. When some of the branches entering the bladder were previously cut, the maximal height of contraction due to nerve stimulation was reduced.

When faradic current of relatively long duration $(3 \mathrm{~min}$.) was applied to the vesical nerve, the bladder responded with prolonged contraction, which obviously corresponded to the tetanic contraction in skeletal muscle. In this case the contraction curve, after it reached a maximum, showed initially small fluctuations at the summit and then gradually declined, although the stimulation was still continued. The whole contraction curve was superposed by fluctuations which became more and more marked as the curve declined.

It must be pointed out that the contractions evoked by nerve stimulation reveal a similar time course as that for automatic contractions except that the former, when they are maximal, exceed the latter in height. This suggests that the auto-rhythmic contractions in bladder might be caused by indirect stimulation, i.e., are neurogenic in origin.

Effects of direct stimulation with A.C. $(60 \sim 1,000$ cycles $)$ resemble in muscle strips that of nerve stimulation in almost all respects. The height of contraction was almost constant irrespective of current intensity except in the neighbourhood of the threshold (fig. 2). With prolonged stimulation, contractions similar to tetanus were obtained. The fluctuations at the summit of a contraction curve showed a somewhat different character according to current intensity. When the contraction was submaximal the fluctuations appeared as relatively large rhythmic contractions which resembled automatic contractions but always ex-
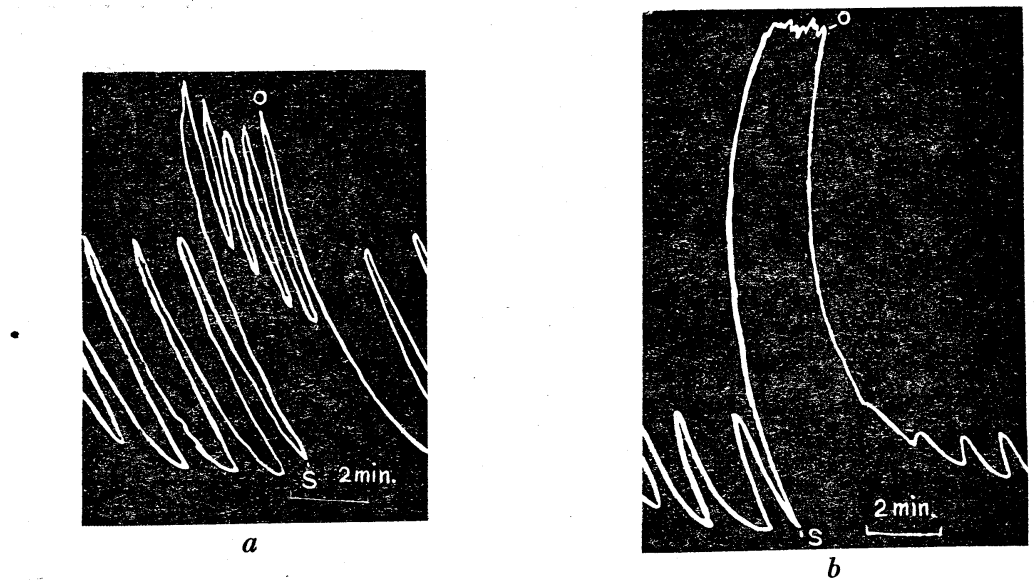

Fig, 3. Responses of muscle strip to A.C. stimulation. $a$ : submaximal stimulation. $b$ : maximal stimulation. Currents flow from $S$ to $O(2 \mathrm{~min}$.). 
ceeded the latter in frequency (fig. $3 a$ ). With a stimulation of supermaximal intensity, the fluctuations at the summit were as a rule much smaller in magnitude, more frequent and also irregular (fig. $3 b$ ). The frequencies of fluctuations, therefore, depended not on the frequency of the stimulating current, but on the current intensity, or rather on the height of contraction.

\section{Response to single break induction shock and its similarity to acetylcholine contracture}

a. Responses to acetylcholine. Responses to acetylcholine are generally referred to as acetylcholine contracture. With increasing concentration the height of shortening increased to a certain extent, beyond which the shortening remained constant notwithstanding further increase in concentration. In case of low concentration $\left(10^{-7}\right)$ the muscle relaxed slowly after initial shortening and regained its original length within about 15 minutes. As the concentration was increased, the relaxation became more and more retarded as well as incomplete. By washing the muscle with Ringer's fiuid, the relaxation was accelerated. As shown

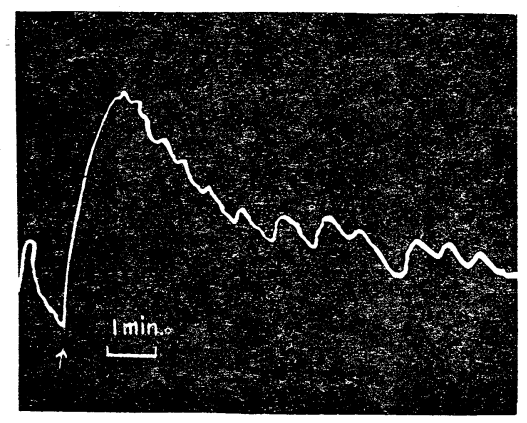

Fig. 4. Effect of acetylcholine $\left(5 \times 10^{-6}\right)$ applied at the point marked with an arrow.

in fig. 4, fluctuations due to automatic contractions were always seen in the relaxation phase, and in some cases also in the contraction phase, so that the myogram assumed the form of a stair.

b. Response to a single break induction shock, As shown in fig. 5 , the response to a single break induction shock was augmented in magnitude continuously as the stimulus was increased. Moreover, the relaxation rate was remarkablely variable according to the intensity of stimulation. The response to a weak stimulus, which was effective to a slight degree only in the later stage of the relaxation phase of automatic contraction, was similar to an automatic contraction both in magnitude and duration. However, as the current intensity

Fig. 5. Relation between contraction height (ordinate) and intensity of stimulation with a single break induction shock (abscissa, coil distance). Circles : stomach. Crosses: bladder.

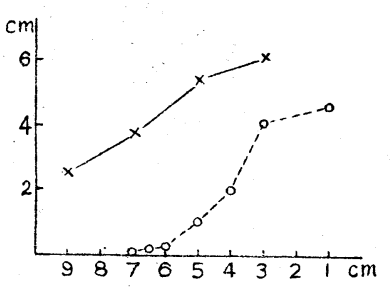


was increased, the current became effective both in the ascending and descending phases of automatic contraction, the contraction height was increased, the latent time reduced and both contraction and relaxation phases were prolonged. Table 1 shows an example. Particularly the retardation of relaxation was marked,

Table 1

\begin{tabular}{c|c|c|c|c}
\hline \hline Coil distance & $\begin{array}{c}\text { Latent } \\
\text { time } \\
\text { sec. }\end{array}$ & $\begin{array}{c}\text { Contraction } \\
\text { height } \\
\text { cm. }\end{array}$ & $\begin{array}{c}\text { Ascending } \\
\text { phase } \\
\text { sec. }\end{array}$ & $\begin{array}{c}\text { Descending } \\
\text { phase } \\
\text { min. }\end{array}$ \\
\hline 9 & 1.04 & 2.5 & 6.6 & 0.37 \\
7 & 0.62 & 3.6 & 7.5 & 0.77 \\
5 & 0.42 & 5.4 & 11.6 & 2.7 \\
3 & 0.16 & 6.1 & 17.3 & $>15$ \\
\hline
\end{tabular}

so that it finally became incomplete, when the current intensity surpassed a certain limit. That is to say, the contraction in these cases was entirely different in nature from those obtainable by the stimulation of the vesical nerve or direct stimulation of muscle with A.C. and also from automatic contractions.

It must be pointed out that these contractions evoked by relatively intense single induction shock show close similarity to acetylcholine contracture in many points, retarded relaxation, superposition of automatic contractions etc. The automatic contractions superposed in the early stage of relaxation were more frequent and smaller in size than those before stimulation. But they became less frequent and intensified as the relaxation proceeded, until finally the original automatic contractions were reestablished (figs. 4 and 6). In some cases automatic
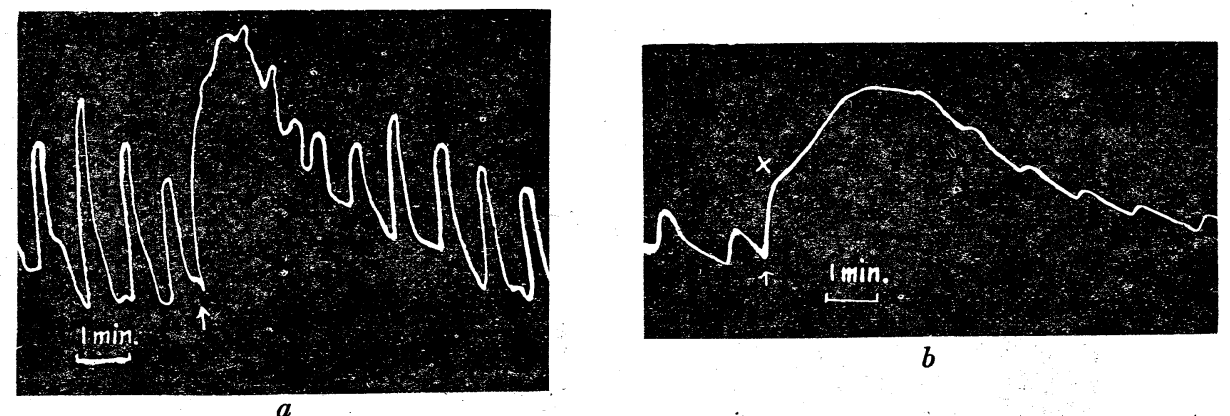

Fig. 6. Responses to single break induction shock. The moment of stimulation is marked by an arrow. $a$ : coil distance, $7 \mathrm{~cm}$. Muscle strip. Bladder. $b:$ another preparation. Notice the flexion point in ascending phase marked with a cross.

contractions were detected even in the ascending phase, as was the case with acetylcholine in low concentration. From these points we may conclude that the slow contraction evoked by single induction shock is a result of direct stimulation of muscular elements and must be considered as the contracture in nature.

Another point worthy of note is that as shown in fig. $6 b$, there appeared often a point of flexion in the ascending phase of a contraction curve evoked by single induction shock. That is, the contraction proceeded at first rapidly 
and then slowly. This suggests that the contraction in these cases involves two different processes; rapid contractile mechanism and a slower one. This point will be discussed later.

\section{Responses to faradic current}

Stimulation with weak faradic current continued for about 10 seconds evoked a contraction similar to that produced by A.C. stimulation. The contraction increased gradually in height with current intensity until it reached a maximum. Also the relaxation time was increased with current intensity and automatic contractions appeared superposed on the descending portion of the myogram. In short, in case of intense faradic stimulation the myogram resembled that obtainable by stimulation with intense single induction shock.

The threshold value for faradic stimulation was always lower than that for single make or break induction shock (latent addition).

\section{Responses to constant current}

a. Response to closure of current. In order to examine the response to closure of a current, constant currents of short duration $(0.05 \sim 0.5 \mathrm{sec}$.) were delivered to the muscle. Weak currents near the threshold evoked a contraction similar to an automatic one, the height of which was extremely variable in accordance with current intensity, from scarcely discernible (ascending phase: about $3 \mathrm{sec}$.) to those which surpassed automatic contractions in magnitude (ascending phase: 15 20 sec.). With further increase in current intensity the response became still more similar to those evoked by single induction shock and the retardation of relaxation became conspicuous.

b. Responses during the current flow. When a constant current of moderate intensity $(8 \sim 10 \mu \mathrm{A})$, which corresponds to a threshold intensity of $0.5 \mathrm{sec}$. duration, flows for a longer time, no effect of current flow is detectable after the initial make-response is over, and there appear ordinary automatic contractions even during the current flow. A slightly more intense current, however, which could produce a phasic contraction as large as an automatic one when applied for a
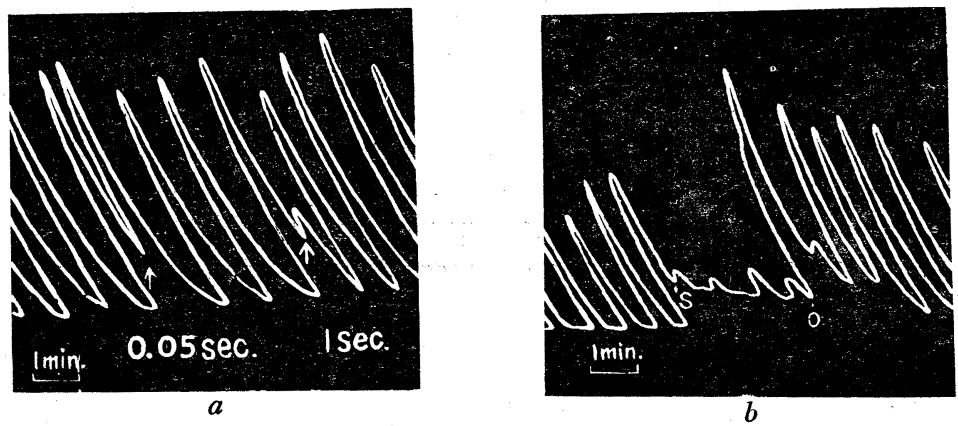

Fig. 7. Responses to constant current.

$a$ : Suppression of make-response by prolonged flow of the same current. Constant currents

$(50 \mu \mathrm{A})$ were delivered for $0.05 \mathrm{sec}$. and $1 \mathrm{sec}$. respectively.

$b$ : Suppression of automatic contractions during the flow of constant current (from $S$ to $0.50 \mu \mathrm{A}, 3 \mathrm{~min}$.$) . The same preparation as in a$. 
short period $(0.05 \sim 0.5 \mathrm{sec}$.), rather depressed the make-response when it was made to flow for a longer period (1 180 sec.); initial contraction was decreased in magnitude and the muscle was kept in a slightly contracted state during the current flow, showing small automatic contractions of the same frequency as before stimulation (fig. 7). In case of a current intensity of about $100 \mu \mathrm{A}$ the inhibition of initial make-response was produced as before and moreover automatic contractions were abolished during the current flow. With still stronger current $(150 \sim 300 \mu \mathrm{A})$, a contracture developed gradually during the current flow.

c. Response to the opening of current. When weak constant current was opened during make-response, no trace of break-response was detectable. Breakresponse which resembled an automatic contraction was obtained only when a relatively strong current was opened after protracted flow. The break-contraction was rather small, when the current had been so intense that a contracture had developed considerably during the current flow. Thus the threshold for breakresponse was, as a rule, higher than that for make-response.

The above mentioned responses to constant current were usually observed in a bladder in a relatively low tonic state. The responses to constant current are extremely variable in accordance with the state of preparation. These points will be dealt with in succeeding communication.

\section{Responses to unidirectional pulse current}

As mentioned above, the stimulation with A.C. $(60 \sim 1,000$ cycles $)$ of considerable intensity $(100 \sim 300 \mu \mathrm{A})$ evoked a maximal contraction, which relaxed rapidly as soon as the current was opend and showed no influence on succeeding automatic contractions. When, however, the current was made an unidirectional pulse current by inserting a vacuum tube in the stimulation circuit, the muscle responded in an entirely different manner, i.e., a prolonged contraction which rather resembles those evoked by intense induction current or acetylcholine. Fig. 8 shows an example. Myograms $a$ and $c$ are contractions evoked by A.C.
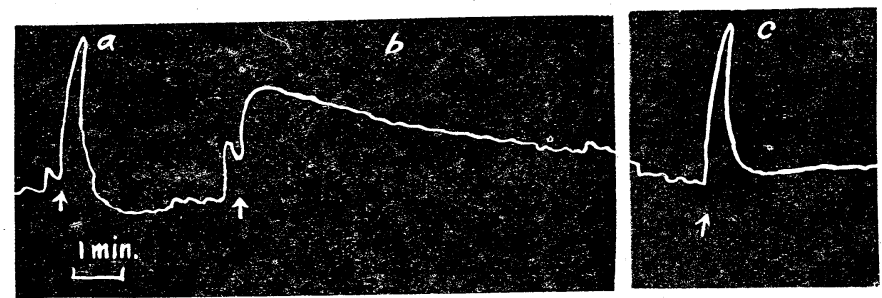

Fig. 8. Responses to A.C. and unidirectional pulse current. $a$ and $c$ are responses to A.C. before and after stimulation with unidirectional pulse current. Response to the latter indicated by $b$. The moments of stimulation are marked with arrows. Current duration: 1 sec.

(1,000 cycle), while $b$ represents the contraction after the current was made unidirectional. The latter was always smaller in height and retarded in relaxation. Even when the amplitude of unidirectional pulse current was doubled to equalize that of the A.C. compared, the contractions produced were always less in 
height than A.C. contractions, while retarded relaxation became more marked. The retardation in relaxation became more marked also with increase in frequency.

From these results the conclusion may be drawn that the unidirectionality of stimulating current is one of the important factors which give rise to prolonged contraction.

\section{The propagation of contractions}

Using the three-portions-method (for details see Kawabata (5)) an investigation was made whether the contractions evoked by various agents at one end portion of the muscle strips were able to propagate to the other end.

a. Chemical contractures, i.e., shortenings produced by acetylcholine $\left(10^{-5}\right)$, $\mathrm{BaCl}_{2}(0.2 \%)$ or $\mathrm{KCl}(0.3 \%)$ at one end portion could not propagate to the other end portion.

b. Contractions evoked by single induction shock or constant current in one end were also not able to propagate to the other end. On the other hand the contractions evoked by A.C. stimulation or by faradic current at one end portion could be conducted to the other end under favourable conditions. Despite some test experiments carried out on the influences of temperature, $\mathrm{Ca}$ content of saline etc, the conditions necessary for propagation could not be determined.

\section{Chronaxie}

The measurement of chronaxie on detrusor muscle is attended by many unavoidable difficulties, namely, the presence of irregular automatic contractions, complicated responses to D.C., as stated in section 4, etc, which make the threshold measurement uncertain, especially in case of stimulation with rheobasic strength. The obstacles, however, can be reduced by using a narrow muscle strip, which shows regular automatic contractions. The threshold values for varying current durations measured on 7 preparations coincided well with each other and in all cases the observed values

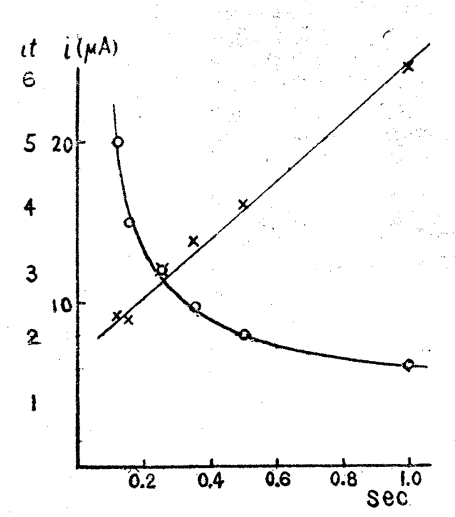

Fig. 9. Strength-duration curve of bladder muscle. $\left(24^{\circ} \mathrm{C}\right)$ fitted considerably well for Weiss' formula. In fig. 9 the observed values in one case are reproduced by circles and crosses, while the curve $(i \cdot t)$ and the straight line $(i t-t)$ in the figure represent theoretical values which were calculated, under the assumption

Table 2.

\begin{tabular}{c|c|c}
\hline No. & $\begin{array}{c}\text { Rheobase } \\
\mu \mathrm{A}\end{array}$ & $\begin{array}{c}\text { Chronaxie } \\
\text { sec. }\end{array}$ \\
\hline 1 & 5 & 0.40 \\
2 & 3 & 0.35 \\
3 & 5 & 0.35 \\
4 & 5 & 0.35 \\
5 & 5 & 0.40 \\
6 & 4.5 & 0.36 \\
7 & 5 & 0.40 \\
\hline \multicolumn{3}{|c}{ (Temperature : $20^{\circ} \sim 25^{\circ} \mathrm{C}$.) }
\end{tabular}


that the Weiss' formula is valid, from rheobase and chronaxie, both obtained by applying the method of least squares to the observed values in 7 cases (table 2).

As expected, the excitability for constant current of the muscle strip showing regular automatic contractions altered rhythmically in accordance with the mechanical cycle. The recovery of excitability proceeded with relaxation, but as a rule it did not attain a constant level, until the next automatic contraction occurred. In the above experiments the measurements were made with test currents delivered to the muscle at a moment when $80 \%$ of the mean automatic cycle had elapsed, so that the muscle was found in an almost completely relaxed state.

To avoid the ambiguity in effectiveness of test current due to automatic contractions, some experiments were performed on perparations showing no automatism at low temperature $\left(0^{\circ} \mathrm{C}\right.$.). Fig. 10 represents a result of a typical

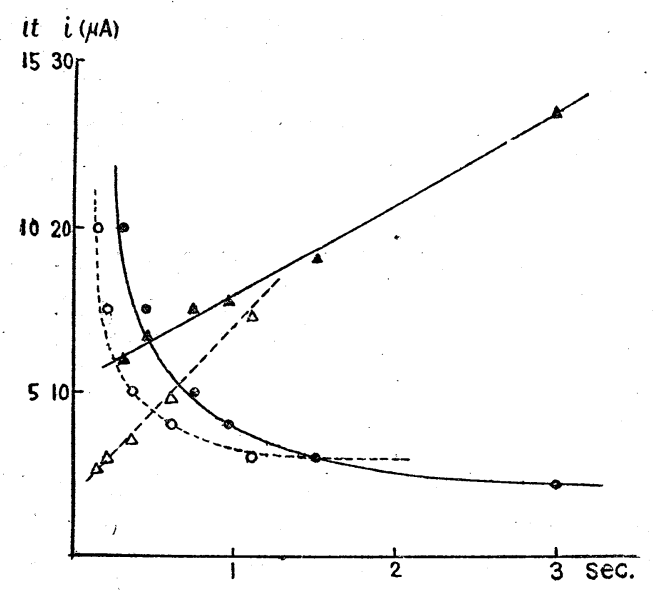

Fig. 10. Strength-duration curves measured at $0^{\circ} \mathrm{C}$. (solid circles) and at $26^{\circ} \mathrm{C}$. (empty circles). The $i t-t$ relations are represented by solid and empty triangles respectively.

experiment in comparison with that obtained when the muscle was returned to room temperature. In the cold, the rheobase was lowered and the chronaxie was markedly lengthened to $2 \sim 3 \mathrm{sec}$.

The muscle strips cut out of the stomach showed in most cases no automatic contraction even at moderate room temperature. The rheobase obtained was $4 \sim 5 \mu \mathrm{A}$ and the chronaxie $1 \sim 2 \mathrm{sec}$.

\section{DISCUSSION}

1. Two kinds of shortening in smooth muscle. It is already known that there are at least two kinds of shortening in smooth muscle, which were designated by previous authors rapid or phasic contraction and slow or tonic contraction (Bozler (2), Riesser (8), Henderson and Roepke (4), Winton (10), Singh (9)). Beside these terms we are accustomed to calling the sustained state of shortening as "tone," when physiologically produced and maintained for several hours, especially as in invertebrate muscles. The sustained contraction is often 
referred to as "contracture," when produced by the action of drugs or ions. Concerning smooth muscles, the term tone is used in an almost synonymous sense to contracture, as indicated by the fact that "Ringer tone" means the same as "Ringer contracture." We know nothing yet what differentiates the two types of contractile mechanisms, designated differently. However, so called tonic contractions may be considered as temporary augmentations of tone or contracture. It seems, therefore, necessary to take into consideration only two types of contraction; the one, rapid or phasic, and the other, a slow tonic contraction or contracture.

The data presented here suggest that the mechanical responses of smooth muscle to electric stimuli consist of two types of contractile mechanism already stated, which are involved in different proportions in every response according to the difference in form of stimulating current. The response which consists almost entirely of the phasic component is the contraction of muscle strip stimulated by alternating current. As an excellent example of the tonic component the contraction evoked by a single induction shock of considerable intensity may be cited, except for the short initial stage which shows a rapid rise. The contractions evoked by various types of electric stimulation can be arranged in the following order, which shows increasing amounts of tonic component involved from left to right;

(Automatic contraction) $<$ vesical nerve stimulation $<$ A.C. stimulation $<$ weak faradic stimulation, weak single induction shock, weak $D$. C. stimulation (make- and break-response only) $<$ unidirectional pulse current $<$ intense single induction shock, intense faradic stimulation, intense D.C. stimulation.

Phasic contractions appear also superposed on tonic contraction, so the automatic contractions or tetanus-like rapid shortening due to A.C. stimulation may appear superposed on prolonged tonic contraction evoked by drugs or intense single induction shock. This suggests a different contractile mechanism underlying these two components and, at the same time, seems to justify the above asserted identification of tonic contraction and tone.

2. The propagation of contractions. The problem whether the contractions in visceral smooth muscle possess conductivity in nature is fairly difficult for an experimental approach at solution because of the minute size of the individual muscle fiber and also of the incomplete syncytial structure, whereby a block of conduction might occur due to some unnoticeable slight impairment. However, the data presented in section 6 seem to offer a clue to this problem. That the contractions evoked by A.C. or faradic current at one end portion of a muscle strip are able in some cases to propagate to another end is definite evidence for the capability of phasic contraction to be conducted throughout the whole syncytial structure. On the other hand tonic contractions chemically induced and contractions by D.C. or single induction shock which consist mainly of a tonic component are never propagated to the other end. This suggests the inability of tonic contraction to be conducted. It seems permissible to con- 
sider the presence of conductivity as a criterion which differentiates phasic contraction from tonic.

The conduction of phasic contractions may be readily blocked at some less resistant points, probably at some intercellular bridge, under unfavourable conditions. As previously reported, automatic contractions do not in general propagate far from their site of origin (Kawabata, 5). If, however, the automatic contractions can not be conducted at all, the contraction initiated at a pacemaker would not grow so as to spread over a certain, even if limited, area and thus the automatism in toad's bladder would become incomprehensible. In reality, the automatic contractions are abolished completely when a D.C. of suitable intensity flows through the muscle, which seems, as discussed below, to restrict the conduction of phasic contraction to a minimal range.

The mechanism of suppression of automatic contraction by a D.C. of suitable intensity is not clear. A possible explanation for this may be sought in electrotonic changes in surface membrane of muscle cells intervening in the pathway of a stimulating current. In relation to this the phenomenon shown in fig. $7 a$

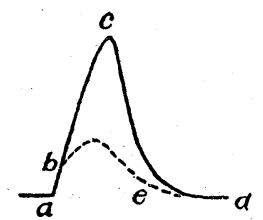

Fig. 11 is worth consideration. Namely a contraction ( $a b c d$ in fig. 11) resembling automatic contraction, evoked by D.C. when it passed through the muscle for $0.05 \sim 0.5 \mathrm{sec}$., was markedly suppressed and showed the time course as abe when the current duration was made longer. This phenomenon is probably accountable to a block in conduction of an initial phasic component by a polarizing effect of D.C.

3. The conditions necessary for the occurrence of two kinds of contraction. The fact that the ratio of two kinds of shortening involved in a response of muscle to electric stimuli varies considerably in accordance with the difference in current form, suggests that different conditions are necessary for the occurrence of these two contractions. As to phasic contraction the circumstances may be considered similar to those necessary for ordinary nervous impulse or contraction in skeletal muscle, since the strength-duration curve measured by employing phasic contraction as an indicator satisfies well the Weiss' formula. On the contrary, tonic contractions have much to do with the quantity of an unidirectional current flow, namely with its duration and intensity. The importance of the intensity factor is exhibited most clearly in the effect of intense single induction shock. Of course the magnitude of tonic contraction is by no means directly proportional to electric quantity. Precise determinations of these relations depend on further research.

In has been reported by Grützner (3) that during the flow of constant current the visceral muscle shows sustained contraction localized in the cathodal region, whereas the muscle under the anode relaxes. These phenomena were recently verified by Masai (7) in our laboratory on retractor pharyngis of snail. Kuffler (6) found also in skeletal muscle, that the relaxation of chemically produced contractures can be effected at the anode of constant currents. In view of this, the above mentioned tonic contraction due to D.C. must be regarded as an algebraic sum of contraction and extension in both polar regions, and conse- 
quently a manifestation that the contraction in the cathodal region surpasses the extension in the anodal region. In the present case we must assume the existence of multitudes of virtual cathodes and anodes in the muscle preparation, because we can obtain only uniform contraction of muscle even if the actual electrodes are situated at both ends of the muscle strip. This assumption is also in accord with the concept of attributing the inhibitory effect of D.C. to electrotonic changes which the current might produce in surface membranes of each cell.

As to phasic contractions, the problem of applicability of the all-or-none law to single muscle fiber lies beyond the scope of the present research. As to tonic contractions, however, the law is no doubt untenable, because the tonic contraction produced by a D.C., which is strong enough to inhibit automatic contractions may be regarded as a sum of contractions in each individual muscle fiber but yet shows increase in magnitude with current intensity.

4. The problem of direct and indirect stimulation. Whether the smooth muscle fiber itself has irritability to electric currents and is able to respond with phasic contraction needs consideration. In view of the long chronaxie value obtained, phasic responses in toad's bladder may be regarded as an effect of direct stimulation of electric currents on muscular elements. For the chronaxie of frog's nerve fiber, even the most sluggish C-fiber, is shorter than 5 msec. (Bishop and Heinbecker, 1). As to the chronaxie of toad's C-fiber we have at present no data availabe, and although the chronaxie values of toad's tissues are always somewhat longer compared to those of frog's corresponding tissues, the present results on toad's bladder are too large to be attributed to nervous elements.

Tonic contractions are doubtlessly results of direct stimulation of muscular tissue, because the stimulation of vesical nerve can arouse, at least primarily, only phasic contractions.

\section{SUMMARY}

1. Toad's bladder responds to various types of electric current with phasic and tonic contractions. The response to indirect stimulation delivered to the vesical nerve consists almost exclusively of phasic contractions. Responses due to direct stimulation consist of both phasic and tonic components contained in different proportions depending on the current form. Response to A.C. stimulation consists, alike indirect stimulation, mainly of phasic components, while those to intense single induction shock and intense D.C. are almost exclusively tonic. When an A.C. is made into an unidirectional pulse current by passing through a vacuum tube, the response becomes strikingly tonic.

2. Phasic contractions can be propagated in nature, and are actually propagated to a certain degree according to the nature of the tissue. Conditions necessary for evoking phasic contractions are considered qualitatively similar to those of nervous impulses or twitches in skeletal muscle.

3. Tonic contraction is nothing more but a temporary change in grade of 
contracture or tone. It can not be conducted and does not show the all-or-none relation. Its occurrence has some relation to the duration of unidirectional current flow, as well as to current intensity.

4. Weiss' formula applies considerably well with strength-duration curve measured by taking phasic contractions as indicators. Rheobases were $3 \sim 5 \mu \mathrm{A}$, chronaxie $0.35 \sim 0.4 \mathrm{sec}$. at $20^{\circ} \sim 25^{\circ} \mathrm{C}$.

The author wishes to thank Prof. T. Otani for helpful suggestions and criticism during the course of this investigation.

\section{REFERENCES}

1. Bishop, G. H. AND Heinbecker, P. Amer. J. Physiol. 94 : 170, 1930.

2. BOZLER, E. Z. vergl. Physiol. $7: 379,1928$.

3. GRUUTZNER, P. Ergebn. Physiol. 3 : 12, 1904.

4. Henderson, V. E. AND Roepke, M. H. J. Pharmacol. 51 : 97, 1934.

5. Kawabata, G. Jap. J. Physiol. 1: 233, 1951

6. KufFleR, S. W. J. Neurophysiol. 8 : 113, 1945.

7. MASAI, S. Unpublished.

8. RIESSER, O. Ergebn. Physiol. 38: 131, 1936.

9. Singh, I. J. Physiol. $92: 241,1938$.

10. Winton, F. R. J. Physiol. $88: 492,1937$.

The expenses of this work were defrayed by a grant from the Ministry of Education. 